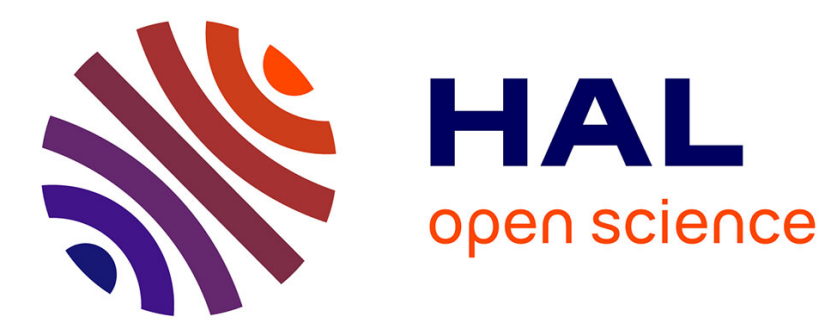

\title{
Heuristiques d'ordonnancement en deux étapes de graphes de tâches parallèles
}

Tchimou N'Takpé

\section{To cite this version:}

Tchimou N'Takpé. Heuristiques d'ordonnancement en deux étapes de graphes de tâches parallèles. Revue des Sciences et Technologies de l'Information - Série TSI : Technique et Science Informatiques, 2009, 28 (1), pp.75-99. inria-00125269v6

\section{HAL Id: inria-00125269 https://hal.inria.fr/inria-00125269v6}

Submitted on 7 Nov 2008

HAL is a multi-disciplinary open access archive for the deposit and dissemination of scientific research documents, whether they are published or not. The documents may come from teaching and research institutions in France or abroad, or from public or private research centers.
L'archive ouverte pluridisciplinaire HAL, est destinée au dépôt et à la diffusion de documents scientifiques de niveau recherche, publiés ou non, émanant des établissements d'enseignement et de recherche français ou étrangers, des laboratoires publics ou privés. 


\section{Heuristiques d'ordonnancement en deux étapes de graphes de tâches parallèles}

\section{Tchimou N'takpé}

Nancy Université / LORIA - UMR 7503

Campus Scientifique - BP 239 F-54506 Vandoeuvre-lès-Nancy Cedex

Tchimou.Ntakpe@loria.fr

RÉSUMÉ. L'ordonnancement d'applications parallèles représentées par des graphes de tâches consiste à trouver l'ensemble de processeurs sur lequel chaque tâche doit être exécutée afin de minimiser le temps d'exécution de ces applications tout en exploitant rationnellement les ressources. Alors que la plupart des algorithmes d'ordonnancement de graphes de tâches parallèles visent des grappes homogènes, cet article montre la nécessité d'avoir de tels algorithmes pour des agrégations de grappes de calcul qui sont de plus en plus répandues et qui peuvent permettre de déployer des applications parallèles à des échelles sans précédents. Nous proposons des améliorations d'une heuristique d'ordonnancement de tâches parallèles en milieu homogène. Ensuite, nous l'adaptons au cas de plates-formes hétérogènes de type grappe hétérogène de grappes homogènes.

ABSTRACT. While most parallel task graph scheduling research has been done in the context of single homogeneous clusters, heterogeneous platforms have become prevalent and are extremely attractive for deploying applications at unprecedented scales. In this paper we address the need for scheduling techniques for parallel task applications for heterogeneous clusters of clusters by proposing a method to adapt existing parallel task graph scheduling heuristics that have proved to be efficient on homogeneous environments. Before adapting that heuristic to heterogeneous platforms, we propose some improvements for homogeneous platforms

MOTS-CLÉS : Heuristiques d'ordonnancement, tâches parallèles, DAGs, grappe de grappes KEYWORDS: Heterogeneous scheduling, parallel tasks, DAGs, cluster of clusters

RSTI - TSI. Volume $28-\mathrm{n}^{\circ} 1 / 2009$, pages 75 à 99 


\section{Introduction}

Une approche récente permettant de pallier la demande croissante en mémoire et en ressources de calcul des applications parallèles consiste à agréger des grappes de calcul existantes soit au sein d'une seule institution, soit réparties entre plusieurs institutions. Il s'agit souvent de grappes de tailles variables dont les capacités peuvent être différentes en fonction des technologies présentes au moment de leur installation. Ces plates-formes composées de plusieurs grappes de stations de travail (Anderson et $a l ., 1995)$ sont à la fois attractives parce qu'elles offrent une importante puissance de calcul, et un défi pour les chercheurs du fait de leur hétérogénéité.

Une des méthodes qui permet d'exploiter la puissance de calcul ainsi disponible est de combiner les parallélismes de tâches et de données présents dans les applications scientifiques. Ces applications peuvent alors être modélisées par des graphes de tâches parallèles. De manière informelle, une tâche parallèle est une tâche qui contient des opérations élémentaires, typiquement une routine numérique ou des boucles imbriquées, qui contiennent suffisamment de parallélisme pour être exécutées par plus d'un processeur. Dans cet article, nous considérons un certain type de tâches parallèles : les tâches modelables (Turek et al., 1992). Ce sont des tâches parallèles pouvant s'exécuter sur un nombre quelconque de processeurs. Ce nombre n'est pas fixé a priori mais est déterminé avant l'exécution et ne varie pas ultérieurement.

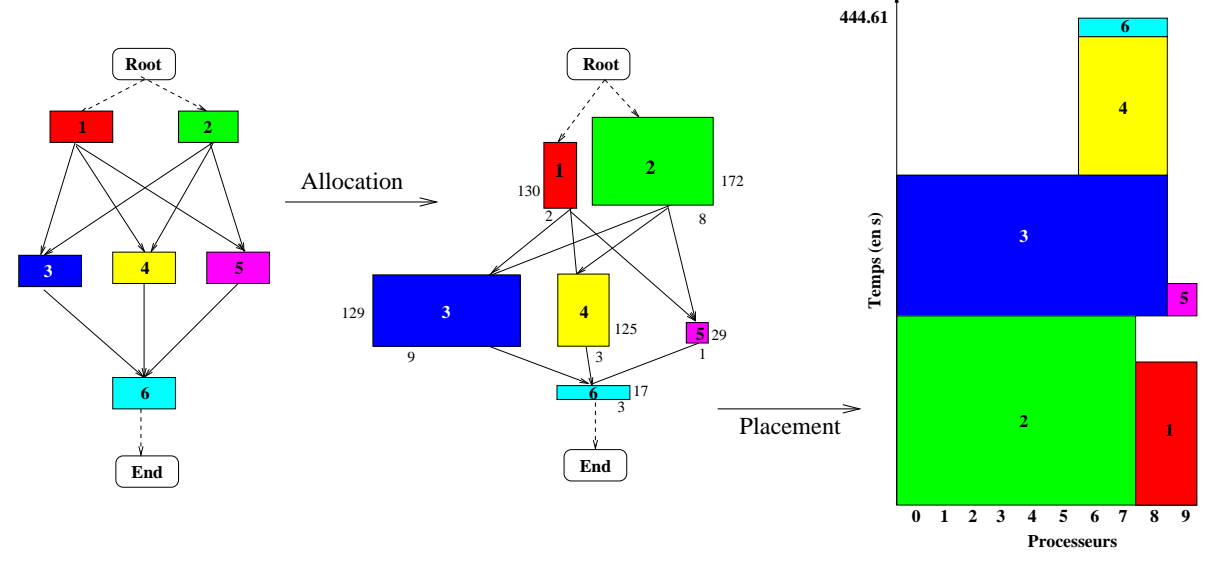

Figure 1. Exemple d'ordonnancement en deux étapes d'un graphe de tâche synthétique sur une grappe homogène de 10 processeurs

La figure 1 illustre le problème de l'ordonnancement de tâches parallèles qui consiste dans un premier temps à trouver le bon nombre de processeurs à allouer à chaque tâche lors de la phase d'allocation. Ensuite il faut déterminer quels sont les processeurs sur lesquels exécuter les différentes tâches lors de la phase de placement. Notons dans cet exemple qu'à l'issue de la phase d'allocation, chaque tâche peut être représentée par une boîte dont la largeur correspond à l'allocation et la hauteur à une 
estimation de son temps d'exécution. Par exemple, 3 processeurs ont étés alloués à la tâche 4 , ce qui conduit à un temps d'exécution de 125 secondes. Les algorithmes d'ordonnancement de tâches parallèles peuvent être répartis en deux catégories. La première est constituée d'algorithmes où la phase d'allocation et la phase de placement sont indissociables. Ce sont les algorithmes d'ordonnancement en une étape (Boudet et al., 2003). La seconde catégorie regroupe les algorithmes d'ordonnancement en deux étapes (Radulescu et al., 2001a; Radulescu et al., 2001b; Ramaswamy et al., 1997; Rauber et al., 1998) où l'allocation et le placement sont séparés en deux procédures distinctes.

De nombreuses études ont été réalisées pour l'ordonnancement de graphes de tâches parallèles dans le cas de plates-formes homogènes (Lepère et al., 2002; Radulescu et al., 2001a; Radulescu et al., 2001b; Ramaswamy et al., 1997; Rauber et al., 1998). Or les plates-formes hétérogènes sont de plus en plus répandues et très attrayantes car elles peuvent permettre de déployer des applications parallèles à des échelles sans précédents. Il est donc nécessaire de développer des heuristiques d'ordonnancement pour de tels systèmes hétérogènes. Une première approche a consisté à adapter une heuristique d'ordonnancement de tâches séquentielles sur plates-formes hétérogènes au cas de tâches parallèles (Casanova et al., 2004). Dans cet article nous nous intéressons à une approche complémentaire consistant à modifier un algorithme d'ordonnancement de tâches parallèles en milieu homogène (Radulescu et al., 2001b) et à prendre en compte l'hétérogénéité des ressources.

Les contributions de cet article sont : (i) apporter des améliorations à l'heuristique choisie dans le cas de plates-formes homogènes, aussi bien dans sa procédure d'allocation que dans sa procédure de placement; (ii) utiliser un nouveau concept de virtualisation des plates-formes qui permet de gérer plus facilement les allocations de ressources en milieu hétérogène; (iii) introduire une nouvelle méthode de placement fondée sur l'idée de l'heuristique sufferage (Maheswaran et al., 1999); (iv) et, définir une méthode d'évaluation par simulation de nombreux scénarios.

Dans la section suivante, nous présentons les modèles de plates-formes et d'applications utilisés. Nous décrirons ensuite notre méthodologie d'évaluation (section 3) puis nous présenterons quelques travaux reliés qui déboucheront sur la formalisation du problème dans la section 4. Après avoir apporté quelques améliorations à l'heuristique d'ordonnancement en milieu homogène présentée dans (Radulescu et al., 2001b) (section 5), nous adapterons l'une des heuristiques obtenues aux plates-formes hétérogènes dans la section 6 . Enfin, la section 7 nous permettra de conclure cet article.

\section{Modèles de plates-formes et d'applications}

Dans cet article, nous considérons des agrégations hétérogènes de grappes homogènes. Ces plates-formes sont représentatives de certaines infrastructures de grilles de calcul réparties entre différentes institutions, qui disposent généralement chacune de grappes homogènes. Nous avons donc $C$ grappes et chaque grappe $C_{k}$ contient $P_{k}$ 
processeurs identiques pour un total de $P$ processeurs sur la plate-forme. Les vitesses des processeurs et les caractéristiques des réseaux locaux ne sont pas nécessairement les mêmes entre les différentes grappes. La figure 2 montre la structure de nos platesformes. Les processeurs d'une même grappe sont reliés entre eux à travers un commutateur (Switch). Les grappes sont reliées par le biais d'une passerelle à un lien réseau très haut débit (dorsale) commun.

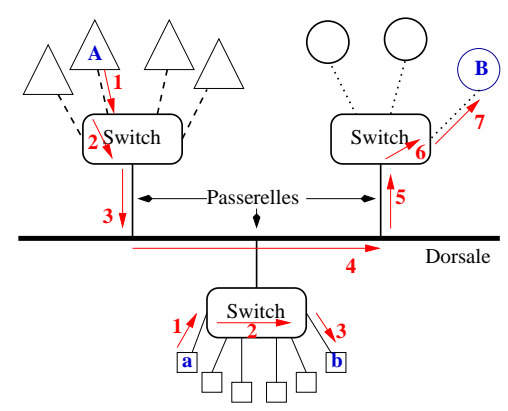

Figure 2. Modèle de plate-forme avec les routes entre deux processeurs A et B localisés sur deux grappes différentes et entre deux processeurs a et b de la même grappe

Une application parallèle peut être modélisée par un graphe acyclique orienté (DAG) $\mathcal{G}=(\mathcal{N}, \mathcal{E})$ où $\mathcal{N}=\left\{t_{i} \mid i=1, \cdots, N\right\}$ est un ensemble de $N$ nœuds (ou tâches) et $\mathcal{E} \subset\left\{e_{i, j} \mid(i, j) \in\{1, \cdots, N\} \times\{1, \cdots, N\}, i<j\right\}$ est un ensemble de $E$ arcs. Les nœuds représentent les tâches parallèles et les arcs définissent les relations de précédence (dépendances de flots ou de données) entre les tâches. On associe à chaque arc $e_{i, j}$, la quantité de données que la tâche $t_{i}$ doit transférer à la tâche $t_{j}$. Par définition, une tâche d'entrée du graphe n'a aucun prédécesseur et une tâche de sortie est sans successeur. Dans cette étude nous utilisons les nœuds Root et End pour représenter respectivement les tâches d'entrée et de sortie des DAGs. Ce sont deux nœuds fictifs sans coût (de calcul ou de communication) qui facilitent la manipulation des graphes de tâches. Une tâche est dite prête lorsque tous ses prédécesseurs ont terminé leur exécution.

Dans une grappe homogène, le temps d'exécution d'une tâche parallèle $t \in \mathcal{N}$ peut être modélisé par un modèle d'accélération classique. Dans cet article, nous utilisons la loi d'Amdahl (Amdahl, 1967) où le temps d'exécution parallèle est donné par :

$$
T(t, p(t))=\left(\alpha+\frac{1-\alpha}{p(t)}\right) \cdot T(t, 1)
$$

$p(t)$ étant le nombre de processeurs alloués à $t, T(t, 1)$ son temps d'exécution sur un seul processeur (temps d'exécution séquentiel) et $\alpha$ sa portion non parallélisable.

Ce modèle d'accélération ayant été conçu pour prédire le temps d'exécution d'une application parallèle sur une grappe homogène (en termes de réseaux et de caractéristiques des nœuds de calcul), nous restreignons l'exécution d'une tâche parallèle à 
l'intérieur d'une grappe. Ce choix est également motivé par le fait qu'en pratique les communications inter-grappes peuvent être très coûteuses. Nous admettons également dans cet article que les processeurs des plates-formes utilisées sont uniformes, c'està-dire que le temps d'exécution séquentiel d'une tâche est inversement proportionnel à la vitesse des processeurs. Dans cet article, la vitesse des processeurs est mesurée en milliards d'opérations en virgule flottante par seconde (Gflops).

Nous définissons enfin $T_{b}(t)$, le bottom level, comme étant la longueur du chemin le plus long depuis la tâche $t$, incluant son propre temps d'exécution, jusqu'à une tâche de sortie quelconque. $T_{b}(t)$ est calculé en faisant la somme des temps d'exécution (prédits) des tâches présentes sur ce chemin.

Le tableau 1 présente les principales notations qui sont utilisées dans cet article. La notion de grappe de référence sera introduite ultérieurement.

\begin{tabular}{|l|l|}
\hline$T_{b}(t)$ & bottom level de la tâche $t$ \\
\hline$T_{C P}$ & longueur du chemin critique du DAG \\
\hline$T_{A}$ & aire moyenne \\
\hline$T_{s}(t)$ & date de début d'exécution d'une tâche $t$ \\
\hline$T_{f}(t)$ & date de fin d'exécution d'une tâche $t$ \\
\hline$C$ & nombre de grappes \\
\hline$P_{r e f}$ & nombre total de processeurs sur la grappe de référence \\
\hline$P_{k}$ & nombre total de processeurs sur la grappe $C_{k}$ \\
\hline$p^{k}(t)$ & nombre de processeurs alloués à la tâche $t$ sur la grappe $C_{k}$ \\
\hline$T^{k}\left(t, p^{k}(t)\right)$ & $\begin{array}{l}\text { temps d'exécution de la tâche } t \text { sur } p^{k}(t) \text { processeurs de la } \\
\text { grappe } C_{k}\end{array}$ \\
\hline$r_{k}$ & $\begin{array}{l}\text { rapport de la puissance d'un processeur de la grappe de } \\
\text { référence sur celle d'un processeur de la grappe } C_{k}\end{array}$ \\
\hline
\end{tabular}

Tableau 1. Liste des principales notations utilisées

\section{Méthodologie d'évaluation}

Pour l'évaluation de nos heuristiques, nous avons recours à des simulations afin d'explorer une large variété de plates-formes et d'applications. L'utilisation de simulations nous permet également de garantir la reproductibilité des expériences et des conditions expérimentales. Pour cela nous utilisons SimGrid ${ }^{1}$ (Casanova et al., 2008), une boîte à outils conçue pour la simulation de grilles de calcul et la mise en œuvre d'applications distribuées. Nous utilisons le modèle de tâches parallèles ptask_L07 qui tient compte du modèle TCP/IP dans les communications dont nous tenons compte dans nos heuristiques pour la prédiction des temps de communications. Dans SimGrid,

1. http ://simgrid.gforge.inria.fr 
pour un flux de communication donné entre deux hôtes distants, la bande passante atteignable sur un lien est le minimum entre la bande passante de ce lien et $\gamma /(2 \times \lambda)$, $\gamma$ étant la taille maximale de la fenêtre TCP (en octets) et $\lambda$ la latence totale (en secondes) entre les deux hôtes. Pour nos expérimentations, la valeur maximale de la fenêtre TCP est fixée à 4Mo comme elle l'est actuellement dans la grille expérimentale grid' $5000^{2}$.

Les simulations sont effectuées sur des plates-formes et des DAGs générés aléatoirement en faisant varier plusieurs paramètres permettant de fixer certaines propriétés.

Les plates-formes générées sont constituées de 1, 2, 4 ou 8 grappes. Le nombre de processeurs de chaque grappe est tiré aléatoirement entre 16 et 128 pour les platesformes dont le nombre de grappes est supérieur ou égal à 2 . Pour les plates-formes à une seule grappe, le nombre de processeurs est tiré entre 16 et 512 . Dans une même plate-forme, les liens intra-grappes sont du Fast Ethernet (débit $=100 \mathrm{Mb} / \mathrm{s}$ et latence $=100 \mu \mathrm{s}$ ) pour les grappes d'indice pair et du Giga Ethernet (débit $=1 \mathrm{~Gb} / \mathrm{s}$ et latence $=100 \mu s$ ) pour les grappes d'indice impair. Ces liens peuvent subir des contentions. La dorsale reliant les grappes entre elles a un débit de $2,5 G b / s$ et une latence de $50 \mathrm{~ms}$. Chaque grappe est connectée à la dorsale via une passerelle ayant une capacité de $1 G b / s$ et une latence de $100 \mu s$.

La borne inférieure des vitesses des processeurs (en G flops) est de 0,25, 0,5, 0,75 ou 1. En ce qui concerne les plates-formes ayant plusieurs grappes, le rapport entre cette borne inférieure et la borne supérieure des vitesses des processeurs de la plateforme, qui constitue le degré d'hétérogénéité, est pris parmi les valeurs 1,2 ou 5 . Nous choisissons ainsi la vitesse des processeurs des différentes grappes de la plateforme en tirant aléatoirement des vitesses entre les bornes inférieure et supérieure. Par exemple si la borne inférieure des vitesses de processeurs est fixée à $0,5 G$ flops et le degré d'hétérogénéité à 5 , alors les vitesses des processeurs sont tirées entre $0,5 G$ flops et $2,5 G$ flops. Pour chaque combinaison de ces paramètres nous générons 10 échantillons dans le cas des plates-formes à une grappe et 5 échantillons pour les autres. Au total, nous avons généré 220 plates-formes dont 40 à une grappe et 180 $(60 \times 3)$ pour les plates-formes comprenant plusieurs grappes.

Les DAGs générés sont constitués de 10, 20 ou 50 tâches parallèles. Nous supposons que les tâches parallèles considérées traitent des nombres réels double précision et que les processeurs ont chacun une mémoire de 1 Go. Les graphes de tâches sont générés en tirant une taille des données $m$, multiple de 1024 (1 Ko), entre les valeurs limites 2048 et 11268 , ce qui correspond au plus à 1 Go d'occupation mémoire par tâche. Ensuite, la complexité de toutes les tâches d'un même DAG est de la forme $a \cdot M$ (ce qui correspond par exemple à la complexité du traitement d'une image de taille $\sqrt{M} \times \sqrt{M}$ ), $a \cdot M \log M$ (tri d'un tableau de $M$ éléments), ou $M^{3 / 2}$ (multiplication de deux matrices de tailles $\sqrt{M} \times \sqrt{M}$ ), ou est tirée au sort parmi ces trois complexités. On a $M=m^{2}$ et $a$ est un nombre tiré aléatoirement entre $2^{6}$ et $2^{9}$ pour tenir compte du fait qu'un algorithme effectue généralement plusieurs opérations. La

2. https ://www.grid5000.fr 
portion non parallélisable de chaque tâche (le $\alpha$ de la loi d'Amdahl) est un nombre aléatoire pris entre 0 et 0,25 . Le volume des transferts est égal à $M$, où $M$ est relatif à la tâche qui génère le transfert.

Quatre paramètres permettent de faire varier la forme des graphes : (i) la largeur $(0,1,0,2$ ou 0,8$)$. Une largeur de 0,8 correspond à un graphe compact avec beaucoup de parallélisme de tâches ; (ii) la régularité entre niveaux $(0,2$ ou 0,8$)$. Dans un graphe peu régulier, la différence entre les nombres de tâches sur deux niveaux consécutifs peut être très importante; (iii) la densité qui caractérise le fait que l'on a plus ou moins de relations de précédence entre les tâches de l'application $(0,2$ ou 0,8$)$; et (iv) la longueur maximale des sauts entre niveaux (1,2 ou 4). Ce dernier paramètre sert à générer des graphes contenant des chemins de longueurs différentes (en nombre de nœuds) entre les tâches d'entrée et de sortie. Pour chaque combinaison de ces paramètres, nous générons 3 échantillons différents, soit 1296 DAGs.

Les simulations étant effectuées sur une large variété de plates-formes et d'applications, il est nécessaire d'utiliser des métriques normalisées pour l'évaluation des performances de nos heuristiques afin d'obtenir des mesures moyennes pertinentes. Nous utiliserons donc les trois métriques suivantes :

le makespan relatif par rapport au meilleur makespan pour une même instance. L'une des mesures les plus utilisées dans l'évaluation des heuristiques d'ordonnancement est le temps de complétion ou makespan. Le makespan est la différence entre la date de début et la date de fin d'exécution d'une application. Autrement dit, c'est le temps d'exécution total de l'application. Le makespan relatif que nous utiliserons dans cet article s'obtient en faisant le rapport du makespan obtenu par une heuristique par le meilleur makespan obtenu par l'une des heuristiques concurrentes pour la même instance (même plate-forme et même DAG). Notons que ce makespan relatif est toujours supérieur ou égal à 1 . Il est égal à 1 si l'heuristique concernée donne le meilleur makespan pour cette instance ;

l'accélération par rapport au meilleur algorithme d'ordonnancement séquentiel (SEQ). Elle mesure le gain en temps d'exécution par rapport à l'ordonnancement des tâches les unes après les autres sur le processeur le plus rapide de la plate-forme considérée;

l'efficacité dont la définition traditionnelle est difficilement généralisable pour les plates-formes hétérogènes. Le travail total $W$ effectué durant l'exécution d'une application est la somme des travaux effectués pour l'exécution de chaque tâche, c'est-à-dire le produit du temps d'exécution de cette tâche (en $s$ ) par la puissance de calcul utilisée (en $G$ flops). SEQ étant l'heuristique qui utilise le plus rationnellement les ressources de calcul, pour un DAG et une plate-forme donnés, nous calculons l'efficacité en faisant le rapport du travail total effectué (par les ressources de calcul) lorsqu' on utilise SEQ par le travail total effectué lorsqu' on utilise l'algorithme à évaluer (cf. équation [2]). Une efficacité élevée (proche de 1) traduit le fait que les ressources de calcul sont rationnellement utilisées dans l'exécution parallèle de l'application. Notons que cette définition de l'efficacité 
ne tient pas compte des éventuels «trous» pouvant se produire dans l'ordonnancement. Notre choix est basé sur le fait que l'ordonnancement peut être implanté afin d'épargner à l'utilisateur le «paiement» des processeurs inutilisés. Sur les plates-formes actuelles, contrôlées par des gestionnaires de ressources, cela peut être réalisé en effectuant plusieurs soumissions ou réservations. L'approche brutale qui consiste à réserver le maximum de processeurs nécessaires pour toute la durée de l'exécution d'une application est très peu économe en ressources pour des DAGs de tâches parallèles.

$$
E_{A L G O}=\frac{W_{S E Q}}{W_{A L G O}}
$$

\section{Travaux précédents}

Le problème de l'ordonnancement de tâches en prenant en compte le coût des communications, est NP-complet au sens fort, même dans le cas ou il existe un nombre infini de processeurs (Chrétienne, 1988). De nombreuses heuristiques ont donc été conçues pour ordonnancer des tâches parallèles. Dans (Dutot, 2005), un algorithme d'ordonnancement de tâches modelables interdépendantes sur une hiérarchie de machines multiprocesseurs est présenté. (Casanova et al., 2004) proposent l'une des rares heuristiques d'ordonnancement de tâches parallèles destinées aux plates-formes hétérogènes (MHEFT). Cette heuristique est obtenue en adaptant un algorithme d'ordonnancement de tâches séquentielles sur plates-formes hétérogènes au cas de DAGs de tâches parallèles. Notre approche complète cette dernière puisque nous partons d'un algorithme d'ordonnancement de graphes de tâches parallèles sur plates-formes homogènes pour l'adapter au cas de plates-formes hétérogènes.

Si certaines études théoriques existent (Lepère et al., 2002), la plupart des algorithmes d'ordonnancement de tâches parallèles (Radulescu et al., 2001a; Radulescu et al., 2001b; Ramaswamy et al., 1997; Rauber et al., 1998) sont des algorithmes en deux étapes et ont été conçus pour des milieux homogènes. La première étape vise à déterminer un nombre de processeurs adéquat pour chaque tâche. Dans la seconde étape, les différents auteurs utilisent des heuristiques de liste pour placer les tâches.

(Ramaswamy et al., 1997) extraient des DAGs à partir de codes séquentiels puis appliquent leur algorithme TSAS (Two Step Allocation and Scheduling). TSAS utilise la programmation convexe, rendue possible grâce à la propriété de posynomialité des modèles de coût choisis, ainsi que par certaines propriétés de leur structure de DAGs. Les fonctions posynomiales sont semblables aux fonctions polynomiales mais elles n'ont que des coefficients positifs et les exposants sont des nombres réels. Une fonction posynomiale peut être transformée en une fonction convexe par un simple changement de variable. Ainsi, la programmation convexe permet aux auteurs d'obtenir en temps polynomial des allocations dans l'espace des réels qu'ils arrondissent ensuite à des nombres entiers. (Rauber et al., 1998) limitent quant à eux leur étude à des graphes construits par compositions séries et/ou parallèles. Dans le premier cas, 
une séquence d'opérations présentant des dépendances de données est placée sur l'ensemble des processeurs. Les tâches de cette séquence sont alors exécutées séquentiellement. Dans le second cas, l'ensemble des processeurs est divisé en un nombre optimal de sous-ensembles, déterminé par un algorithme glouton. Le critère d'optimisation de cet algorithme est la minimisation du temps de complétion de l'ensemble des tâches.

Dans cet article, nous nous intéressons à l'algorithme CPA (Radulescu et al., 2001b) qui est à la fois peu coûteux en termes de complexité et relativement performant aussi bien vis-à-vis du temps de complétion des applications que pour la gestion des ressources. CPA vise à obtenir le meilleur compromis entre la longueur du chemin critique, c'est-à-dire le plus long chemin du graphe en tenant uniquement compte des temps d'exécution des tâches, et l'aire moyenne du diagramme de Gantt. Radulescu et al. remarquent que le temps d'exécution d'une application parallèle peut être approché par sa borne inférieure $T_{p}^{e}=\max \left\{T_{C P}, T_{A}\right\}$, où $T_{C P}$ est la longueur du chemin critique et $T_{A}$, l'aire moyenne :

$$
\begin{aligned}
& T_{C P}=\max _{t \in \mathcal{N}} T_{b}(t), \text { et } \\
& T_{A}=\frac{1}{P} \sum_{i=0}^{N}\left(T\left(t_{i}, p\left(t_{i}\right)\right) \times p\left(t_{i}\right)\right) .
\end{aligned}
$$

Notons que la notion d'aire moyenne définie par les auteurs de CPA a la dimension d'un temps. Elle peut être interprétée comme étant le temps moyen d'utilisation des processeurs.

Le but de CPA est de minimiser $T_{p}^{e}$ au terme de la phase d'allocation. Sachant que $T_{C P}$ diminue tandis que $T_{A}$ croît lorsque le nombre de processeurs alloués aux tâches augmente, on initialise les allocations en partant du cas où $T_{C P}$ est maximal en allouant un processeur à chaque tâche. Ensuite à chaque itération de la procédure d'allocation, on alloue un processeur de plus à la tâche la plus prioritaire jusqu'à ce que l'on obtienne $T_{C P} \leq T_{A}$. Cette tâche la plus prioritaire est celle appartenant au chemin critique et dont le rapport $T(t, p(t)) / p(t)$ diminue le plus significativement si un processeur supplémentaire lui est attribué. Dès qu'elle est vérifiée, la condition d'arrêt de cette procédure d'allocation $\left(T_{C P} \leq T_{A}\right)$ traduit le fait que $T_{p}^{e}$ est proche d'un minimum local ( $T_{p}^{e} \approx T_{C P} \approx T_{A}$ ). La figure 3 présente l'évolution de $T_{C P}$ et de $T_{A}$ en fonction du nombre d'itérations de la procédure d'allocation de CPA appliquée à l'exemple de la figure 1 .

A chaque itération de la procédure de placement, la tâche prête ayant le plus grand bottom level est choisie pour être placée. Lorsqu'une tâche est prête (tous ses prédécesseurs ont terminé leur exécution), l'emplacement des données nécessaires à son exécution est connu. Il est donc alors possible de tenir compte des temps de redistributions de données et déterminer les processeurs qui minimisent la date de fin d'exécution $T_{f}(t)$ de chaque tâche prête $t . T_{f}(t)$ dépend entre autres de la date d'arrivée 
de la dernière donnée nécessaire à l'exécution de $t$ et de la date de disponibilité des processeurs choisis.

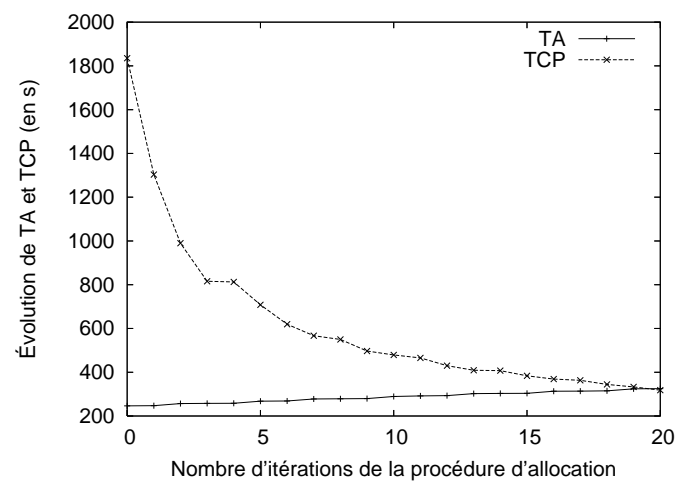

Figure 3. Exemple d'évolution de $T_{C P}$ et $T_{A}$ lors de la procédure d'allocation

Dans (Radulescu et al., 2001b) il a été montré que la complexité de CPA est de l'ordre de $O(N(N+E) P)$. $N, E$ et $P$ représentent respectivement le nombre de nœuds du DAG, le nombre d'arcs du DAG et le nombre de processeurs de la plateforme .

\section{Améliorations de CPA en milieu homogène}

Dans cette section, nous nous plaçons dans le cas où les plates-formes sont constituées d'une seule grappe homogène et nous proposons deux améliorations de l'algorithme original de Radulescu et al., la première porte sur la phase d'allocation et la seconde sur la phase de placement.

\subsection{Nouveau critère d'arrêt dans la procédure d'allocation}

Par expérience, nous avons constaté que le calcul de l'aire moyenne de CPA est moins pertinent lorsque le nombre de processeurs $(P)$ de la plate-forme est beaucoup plus grand que le nombre de tâches $(N)$. En effet, $T_{A}$ converge très lentement vers $T_{C P}$ lorsque le nombre de processeurs de la plate-forme est très grand. Cela débouche sur des allocations contenant un très grand nombre de processeurs. Or plus on alloue de processeurs à chacune des tâches, plus le risque de ne plus pouvoir exécuter en parallèle certaines tâches concurrentes augmente. Il peut donc s'avérer préférable d'arrêter le processus d'allocation plus tôt et donc de déterminer des allocations plus petites afin de profiter au mieux du parallélisme de tâches. Nous proposons pour cela le compromis suivant qui permet d'arrêter plus vite la procédure d'allocation dans le 
cas où le nombre de ressources est très élevé en prenant $\min (P, \sqrt{P \times N})$ au lieu de $P$. Cela nous conduit à redéfinir la notion d'aire moyenne de la façon suivante :

$$
T_{A}^{\prime}=\frac{1}{\min (P, \sqrt{P \times N})} \sum_{i=0}^{N}\left(T\left(t_{i}, p\left(t_{i}\right)\right) \times p\left(t_{i}\right)\right)
$$

Pour $P \gg N$, cette nouvelle définition augmente la pente de croissance de l'aire moyenne. La relation $T_{p}^{e} \approx T_{C P}$ reste toujours valable à la fin de la procédure d'allocation.

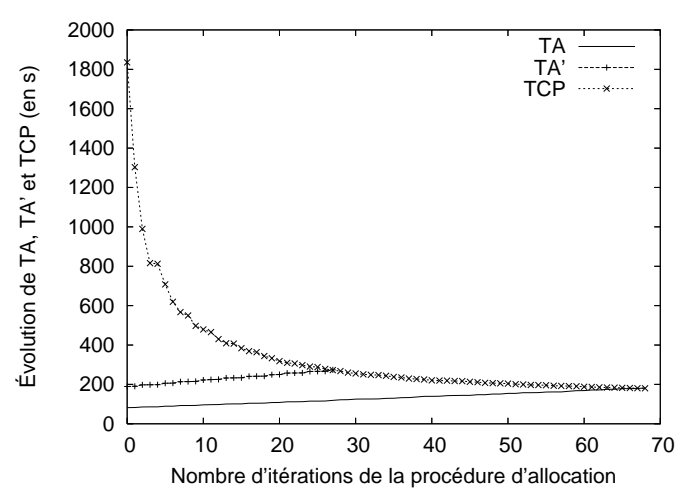

Figure 4. Exemple d'évolution de $T_{A}, T_{A}^{\prime}$ et de $T_{C P}$ dans la procédure d'allocation de CPA et dans la nouvelle procédure d'allocation

La figure 4 montre l'évolution de $T_{C P}$ et de $T_{A}$ en utilisant CPA ainsi que celle de $T_{A}^{\prime}$ dans la nouvelle procédure d'allocation sur le DAG de la figure 1. Dans cet exemple, le nombre de processeurs $(P=30)$ est supérieur au nombre de tâches $(N=6)$. D'où une convergence plus rapide de $T_{C P}$ et $T_{A}^{\prime}$ dans le cas de la nouvelle procédure d'allocation. On note une importante réduction du nombre total de processeurs alloués lorsqu'on utilise la nouvelle procédure d'allocation (33 processeurs $=6$ processeurs alloués dès l'initialisation +27 processeurs supplémentaires) par rapport au nombre total de processeurs alloués avec CPA $(74=6+68)$. Cette réduction des allocations fait passer la longueur du chemin critique de 180, 10 secondes à 272, 58 secondes mais elle permet de mieux profiter du parallélisme de tâches existant dans le DAG. Ainsi, dans la figure 5 qui présente le résultat de l'ordonnancement, nous pouvons observer que l'exécution en parallèle des tâches 1 et 2, puis des tâches 3 , 4 et 5 permet d'obtenir un meilleur temps de complétion par rapport à l'ordonnancement de CPA en plus d'une moindre consommation de ressources. Nous sommes conscients que le compromis que nous venons de définir n'est pas toujours optimal du point de vue du temps de complétion des applications mais il permet surtout de mieux gérer l'utilisation des ressources. En effet, hormis les tâches «entièrement parallélisables », plus l'on alloue de processeurs aux tâches, plus l'efficacité diminue. 


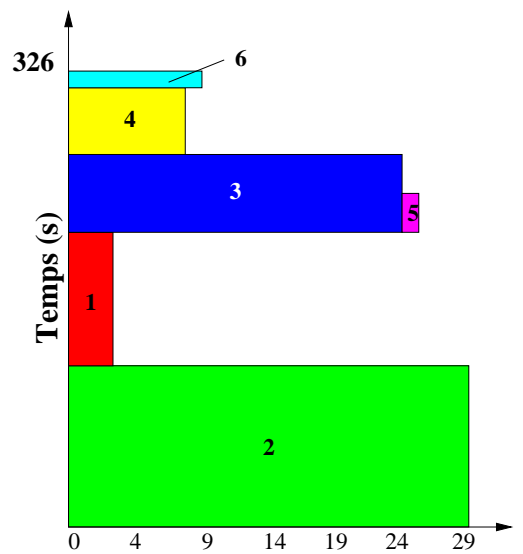

(a)

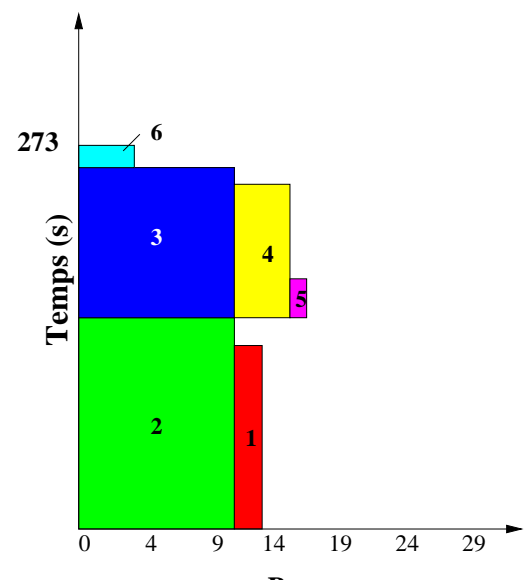

(b)

Figure 5. Ordonnancement du DAG de la figure 1 avec CPA (a) et en utilisant la nouvelle allocation (b) sur une grappe homogène de 30 processeurs

\subsection{Tassage lors du placement}

Comme dans l'heuristique CPA (Radulescu et al., 2001b), les procédures de placement que nous proposons dans cet article sont dynamiques, c'est-à-dire que la décision de placement n'est effectuée qu'une fois la tache concernée prête. L'avantage de cette technique de placement est qu'elle s'adapte plus facilement à des plates-formes dynamiques partagées par de nombreux utilisateurs, contrôlées par des gestionnaires de ressources.

La phase d'allocation et la phase de placement des tâches étant découplées, il peut arriver qu'une tâche prête se mette à attendre qu'une partie des processeurs qui lui sont alloués soient disponibles alors que la majorité des processeurs dont elle aurait besoin le sont déjà. Cette tâche pourrait donc avoir une meilleure date de fin d'exécution si l'on réduisait son allocation de sorte qu'elle puisse démarrer dès la date où elle est prête.

La technique de tassage que nous proposons permet d'éviter cette situation. Dans ce cas il améliore la date de fin d'exécution d'une tâche prête tout en réduisant le nombre de processeurs qui lui sont alloués. La procédure de placement avec tassage se comporte de la manière suivante :

1) à l'instant où la tâche prête la plus prioritaire est choisie, on regarde s'il est possible de débuter son exécution immédiatement avec son allocation initiale, c'est-àdire que le nombre de processeurs disponibles est supérieur ou égal à cette allocation. Dans ce cas, la tâche est placée sur les processeurs disponibles; 
2) si le nombre de processeurs disponibles est inférieur à l'allocation déterminée pour cette tâche, il faut vérifier si elle pourrait terminer son exécution plus tôt en utilisant seulement les processeurs déjà disponibles plutôt qu'en attendant que tous les processeurs qui lui sont alloués soient libres. Si c'est le cas, alors une nouvelle allocation lui est attribuée et la tâche est placée sur les processeurs disponibles. Sinon elle attend qu'il y ait suffisamment de processeurs libres en vue d'être placée selon son allocation initiale.
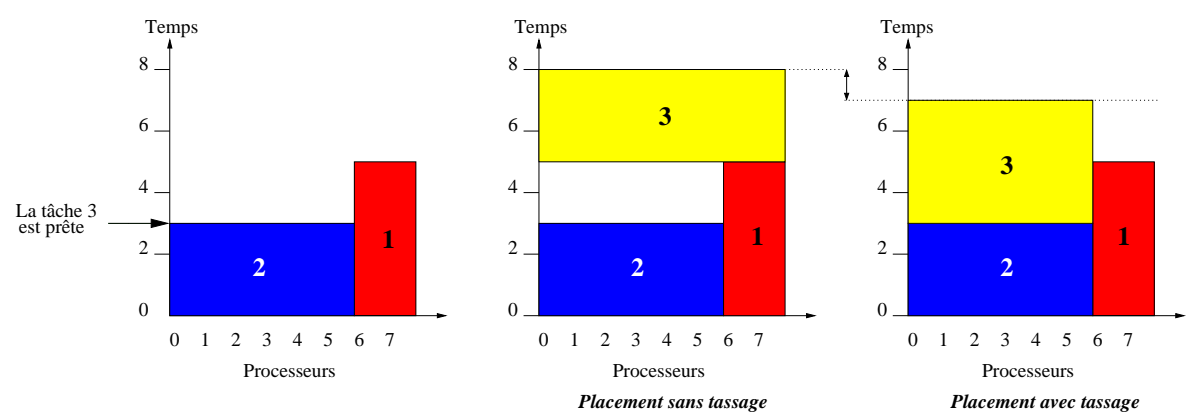

Figure 6. Illustration du placement avec tassage

La figure 6 illustre un exemple de tassage pour une tâche prête. Le fait de réduire l'allocation de la tâche 3 de 8 à 6 processeurs permet d'avoir une meilleure date de fin d'exécution pour cette tâche.

Dans la section suivante, nous comparons les performances obtenues en appliquant chacune des modifications proposées avec la version originale de l'algorithme CPA (Radulescu et al., 2001b) en milieu homogène. Nous regardons également comment l'algorithme se comporte lorsque nous combinons les deux modifications que nous venons de décrire.

\subsection{Evaluation des algorithmes}

La figure 7 compare les moyennes du makespan relatif et de l'efficacité des algorithmes sur l'ensemble des DAGs et pour les plates-formes à une grappe dans les cas suivants :

- utilisation de l'algorithme original (CPA);

- utilisation du tassage pendant le placement;

- utilisation de $T_{A}^{\prime}$ au lieu de $T_{A}$;

- utilisation combinée de $T_{A}^{\prime}$ et du tassage.

Nous observons qu'en moyenne, le tassage permet de réduire le makespan des applications par rapport à CPA. En effet, cette amélioration de l'heuristique de placement permet de réduire les trous dans l'ordonnancement en diminuant légèrement 
l'allocation de certaines tâches. En ce qui concerne l'utilisation de $T_{A}^{\prime}$, non seulement elle permet de gagner de manière significative sur le temps de complétion des applications, mais elle utilise aussi plus efficacement les ressources par rapport à CPA. Cela confirme le fait qu'il est important d'arrêter les allocations suffisamment tôt afin de pouvoir exécuter les tâches prêtes concurrentes en parallèle. Nous observons également que la combinaison des deux améliorations ( $T_{A}^{\prime}$ et tassage) n'améliore que très légèrement les performances par rapport à l'utilisation de $T_{A}^{\prime}$ seule, cette dernière ayant un apport prédominant.
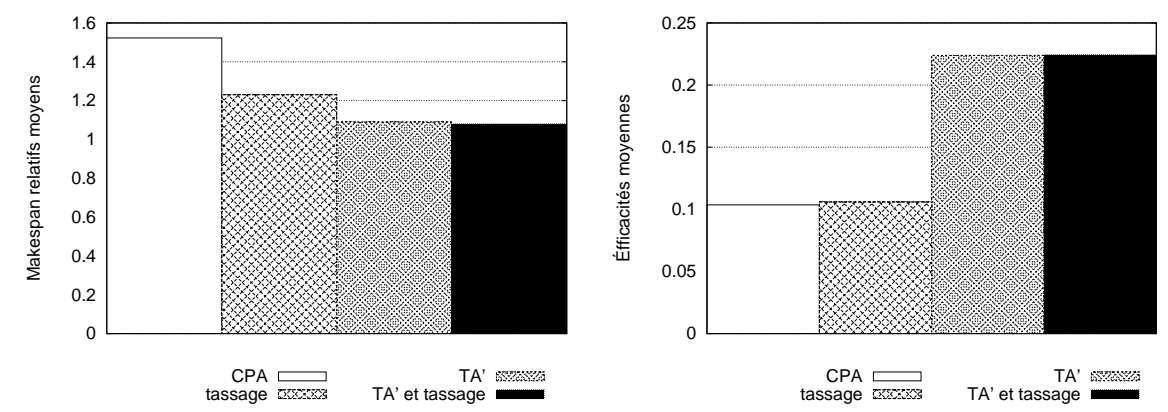

Figure 7. Performances globales sur une grappe

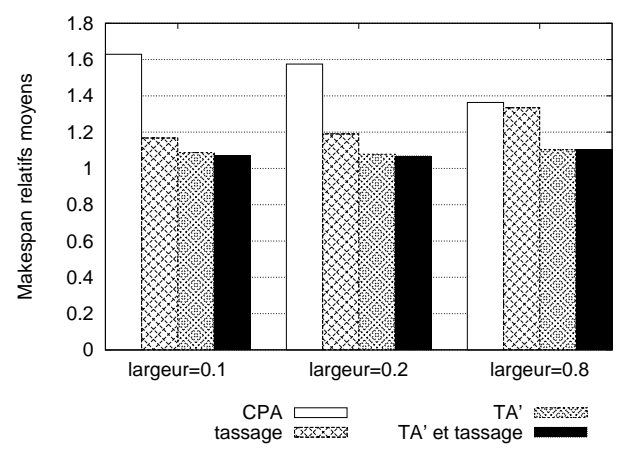

Figure 8. Makespan relatifs moyens en fonction de la largeur des graphes

Lorsque nous regardons l'évolution du makespan relatif des algorithmes en fonction de la largeur des DAGs (figure 8), il apparaît que pour les DAGs peu larges (largeur $\leq 0,2)$ le tassage améliore significativement le makespan par rapport à CPA. Par contre pour les DAGs de largeur 0,8 où il existe de nombreuses tâches pouvant s'exécuter en parallèle, le tassage seul n'est pas suffisant pour réduire sensiblement le makespan par rapport à CPA. Or le fait d'utiliser $T_{A}^{\prime}$ permet d'exécuter plusieurs tâches en parallèles en ayant des allocations réduites. Ainsi l'utilisation de $T_{A}^{\prime}$ permet d'améliorer de manière notoire le makespan par rapport à CPA. 
Dans la section suivante, nous adaptons l'heuristique obtenue en combinant l'utilisation de $T_{A}^{\prime}$ et du tassage au cas des plates-formes hétérogènes. Nous mettrons en place deux heuristiques qui diffèrent par leurs phases de placement.

\section{Adaptation aux plates-formes hétérogènes}

La plate-forme cible étant maintenant constituée de $C$ grappes, notre idée consiste à attribuer, lors de la première phase, une allocation de référence à chaque tâche qui représentera ses $C$ allocations potentielles. Nous définirons comment déterminer le nombre de processeurs à allouer à une tâche sur une grappe en fonction de son allocation de référence dans la section 6.1. Lors de la phase de placement nous retiendrons parmi les allocations potentielles celle qui minimise la date de fin d'exécution d'une tâche prête.

Etant donné qu'il existe désormais plusieurs allocations possibles pour une même tâche, il convient de redéfinir formellement les notions de chemin critique et d'aire moyenne qui déterminent la condition d'arrêt de la phase d'allocation. Pour cela nous introduisons la notion de grappe de référence sur laquelle nous ferons évoluer l'allocation des processeurs. Cette grappe de référence est une plate-forme homogène virtuelle ayant une puissance de calcul cumulée équivalente à celle de l'ensemble de la plate-forme réelle et dont les processeurs ont la plus petite vitesse de la plate-forme initiale. Le nombre total de processeurs contenus dans la grappe de référence est donc :

$$
P_{\text {ref }}=\left\lceil\sum_{k=0}^{C-1} \frac{P_{k}}{r_{k}}\right\rceil
$$

où $r_{k}$ est le rapport de la puissance d'un processeur de la grappe de référence sur celle d'un processeur de la grappe $C_{k}$. L'utilisation de cette grappe homogène virtuelle permet de conserver une faible complexité dans le nouvel algorithme. Notons $p^{r e f}(t)$, l'allocation de référence pour la tâche $t$, c'est-à-dire le nombre de processeurs qui lui serait attribué sur la grappe de référence. L'allocation de référence est définie de sorte que le temps d'exécution effectif de chaque tâche soit relativement proche du temps qu'elle mettrait sur la grappe de référence : $T^{r e f}\left(t, p^{r e f}(t)\right)$. La longueur du chemin critique $\left(T_{C P}\right)$ et l'aire moyenne $\left(T_{A}^{\prime}\right)$ se définissent maintenant par rapport aux allocations de référence :

$$
\begin{aligned}
& T_{C P}=\max _{t \in \mathcal{N}} T_{b}^{r e f}(t), \\
& T_{A}^{\prime}=\frac{1}{\min \left\{P_{\text {ref }}, \sqrt{P_{\text {ref }} \times N}\right\}} \sum_{i=0}^{N}\left(T^{\text {ref }}\left(t_{i}, p^{\text {ref }}\left(t_{i}\right)\right) \times p^{\text {ref }}\left(t_{i}\right)\right),
\end{aligned}
$$

$T_{b}^{r e f}(t)$ étant le bottom level calculé à partir des allocations de référence des tâches. 
Les deux sections qui suivent décrivent les deux étapes des heuristiques que nous avons conçus.

\subsection{Allocation de processeurs}

Comme dans CPA, à chaque itération de la procédure d'allocation, la tâche du chemin critique qui en bénéficie le plus se voit attribuer un processeur supplémentaire. Dans nos algorithmes ce processeur est ajouté à l'allocation de référence. Pour déterminer le nombre de processeurs à allouer à une tâche sur une grappe donnée, d'après son allocation de référence, nous utilisons la loi d'Amdahl. Du fait que nous utilisons un modèle de processeurs uniformes, l'égalité :

$$
T^{k}\left(t, p^{k}(t)\right)=T^{r e f}\left(t, p^{r e f}(t)\right)
$$

pour $k$ fixé, conduit à :

$$
f\left(p^{r e f}(t), t, k\right)=\frac{(1-\alpha) \cdot T^{k}(t, 1)}{\left.T^{r e f}\left(t, p^{r e f}(t)\right)-\alpha \cdot T^{k}(t, 1)\right)}
$$

$f$ étant la fonction qui permet de déduire $p^{k}(t)$, l'allocation de la tâche $t$ sur la grappe $C_{k}$. Puisqu'on ne peut allouer plus de processeurs qu'une grappe n'en contient et que le nombre de processeurs alloués doit être un nombre entier, on en déduit :

$$
p^{k}(t)=\min \left\{P_{k},\left\lceil f\left(p^{r e f}(t), t, k\right)\right\rceil\right\}
$$

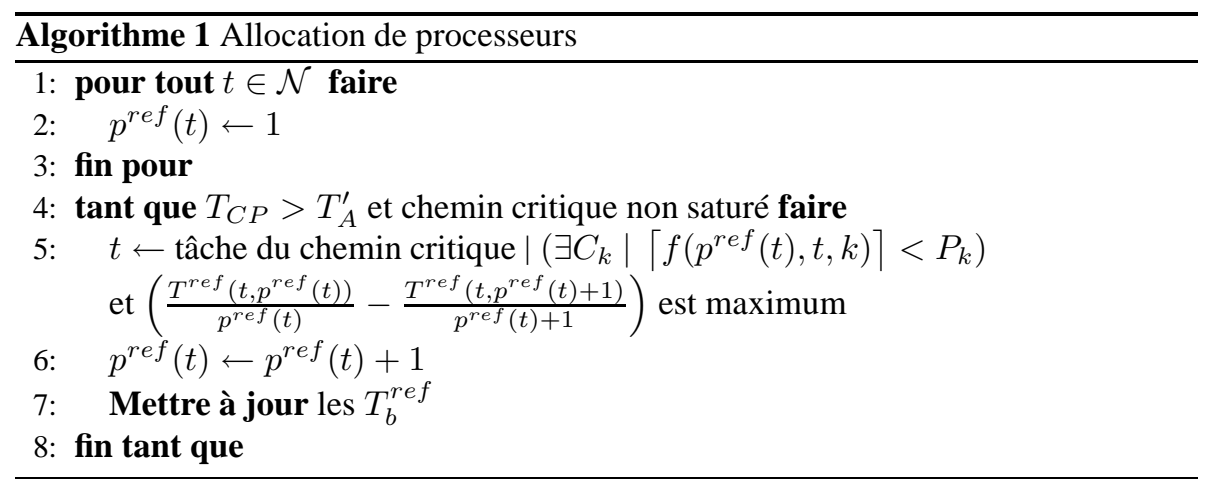

Pour éviter une boucle infinie dans cette procédure, nous définissons une condition d'arrêt supplémentaire qui est la notion de chemin critique saturé. Le chemin critique est dit saturé si les allocations de référence sont telles qu'il est impossible de rajouter des processeurs aux tâches qui composent le chemin critique. Le nombre de processeurs à allouer à chacune des tâches du chemin critique sur toute grappe $C_{k}$ est 
alors le nombre total de processeurs de cette grappe $\left(P_{k}\right)$. Les temps d'exécution des tâches du chemin critique ne peuvent plus être réduits en augmentant leurs allocations de référence, $T_{C P}$ est alors minimal et nous arrêtons la procédure d'allocation dès que cet état est atteint. L'algorithme 1 présente notre version hétérogène de la phase d'allocation.

\subsection{Placement des tâches}

Le placement consiste à attribuer à chaque tâche prête choisie $t$, les processeurs qui lui garantissent la plus petite échéance $T_{f}(t)$. Soit $T_{r}^{k}\left(t_{i}, t_{j}\right)$ le temps nécessaire à la redistribution des données entre une tâche $t_{i}$ qui vient de s'exécuter (sur un ensemble de processeurs connu) et une tâche $t_{j}$ qui lui succède, en considérant son placement sur la grappe $C_{k}$. Ce temps dépend notamment des caractéristiques du réseau, de la quantité des données à transférer et des nombres de processeurs alloués aux tâches $t_{i}$ et $t_{j}$. Soit $T_{m}^{k}(t)$ la date d'arrivée de la dernière donnée d'une tâche prête $t$ sur la grappe $C_{k}$. On a :

$$
T_{m}^{k}(t)=\max _{t_{i} \in \operatorname{Pred}(t)}\left(T_{f}\left(t_{i}\right)+T_{r}^{k}\left(t_{i}, t\right)\right)
$$

où $\operatorname{Pred}(t)$ est l'ensemble des prédécesseurs de $t$. La date à laquelle la tâche $t$ peut effectivement démarrer son exécution est donc :

$$
T_{s}^{k}(t)=\max \left\{\operatorname{dispo}\left(p^{k}(t)\right), T_{m}^{k}(t)\right\}
$$

où $\operatorname{dispo}\left(p^{k}(t)\right)$ est la date à laquelle la grappe $C_{k}$ aura au moins $p^{k}(t)$ processeurs libres.

Une fois les allocations potentielles des tâches définies, nous avons étudié deux politiques différentes pour le placement des tâches.

Dans la première nous adaptons l'algorithme d'ordonnancement de liste de CPA au cas où l'on dispose de plusieurs allocations possibles pour chaque tâche. Comme dans CPA, la tâche prête la plus prioritaire est celle qui a le bottom level le plus élevé. Une fois que cette tâche $t$ est déterminée, nous choisissons l'allocation qui minimise sa date de fin d'exécution :

$$
T_{f}(t)=\min _{k}\left(T_{s}^{k}(t)+T^{k}\left(t, p^{k}(t)\right)\right)
$$

Nous en déduisons $C_{k}$, la grappe sur laquelle son exécution est prévue ainsi que sa date de début d'exécution : $T_{s}(t)=T_{s}^{k}(t)$. La combinaison de la procédure d'allocation et cet algorithme de placement donne lieu à l'heuristique HCPA (Heterogeneous Critical Path and Area-based).

Dans notre seconde heuristique d'ordonnancement, la tâche la plus prioritaire est déterminée selon le principe de l'heuristique sufferage (Maheswaran et al., 1999). Ce 
principe consiste à choisir parmi les tâches prêtes celle qui serait la plus pénalisée s'il lui était attribué sa deuxième meilleure allocation au lieu de la première. Cette tâche est donc celle qui accuse la plus grande différence entre les deux dates de fin d'exécution prédites. Cette heuristique ne tient donc pas compte du chemin critique. Le but de sa mise en œuvre est de voir s'il peut être parfois intéressant d'utiliser des heuristiques de placement autres que celles fondées sur le chemin critique. Nous obtenons ainsi l'algorithme SHCPA (Sufferage-based Heterogeneous Critical Path and Area-based). Ici également, le placement de la tâche prête la plus prioritaire vise à minimiser sa date de fin d'exécution en lui attribuant sa meilleure allocation.

\subsection{Complexité de HCPA et SHCPA}

Soit $K$, le nombre de processeurs maximum qu'on pourrait attribuer à une tâche sur la grappe de référence :

$$
K=\max _{(k, t)}\left\lceil f^{-1}\left(P_{k}, t, k\right)\right\rceil
$$

Dans le pire des cas, il faudrait $K$ itérations pour chaque tâche dans la procédure d'allocation. D'où un total de $K \times N$ itérations de la procédure d'allocation. la complexité du corps de la boucle (choix du chemin critique, calcul de $T_{C P}, T_{b}$, et $T_{A}$ ) est de l'ordre de $O((N+E) C)$. Nous en déduisons la complexité de la phase d'allocation : $O(N(N+E) C \times K)$.

En ce qui concerne la phase de placement, dans le cas de HCPA, les tâches prêtes sont triées par ordre de priorité à l'aide d'une procédure de l'ordre de $O\left(N^{2}\right)$ dans le pire des cas (cas où toutes les tâches du DAG sont prêtes en même temps du premier coup). Pour chaque tâche prête à placer, l'on teste les $C$ allocations possibles. Au total, la procédure de placement de HCPA est de l'ordre de $O(N(N+C))$. Cette complexité est négligeable par rapport à celle de la phase d'allocation. Pour la phase de placement de SHCPA, dans le pire des cas, les $N$ tâches sont prêtes en même temps et la détermination de la $\mathrm{i}^{\text {eme }}$ tâche la plus prioritaire a un coût de l'ordre de $C \times(N-i)$. En effet, on examine les $C$ allocations possibles pour chacune des $N-i$ tâches prêtes pas encore placées. La complexité de la procédure de placement de SHCPA est donc de l'ordre de $C \times N^{2}$. Cette complexité peut également être négligée par rapport à celle de la phase d'allocation.

Il en découle que la complexité de HCPA et SHCPA est de l'ordre de

$$
O(N(N+E) C \times K)
$$

Dans le cas d'une plate-forme composée d'une unique grappe homogène, la complexité de nos heuristiques est identique à celle de CPA puisque $C=1$ et $K=P$. Dans le cas d'une plate-forme hétérogène comprenant plusieurs grappes, il convient d'exprimer le facteur $C \times K$ en fonction du nombre total de processeurs $P$. Soit $h$ 
le degré hétérogénéité de la plate-forme qui correspond au rapport entre la vitesse du processeur le plus rapide et celle du processeur le plus lent. D'après l'équation [6] le nombre de processeurs de la grappe de référence est inférieur à $h \times P$, puisque $1 / r_{k} \leq h, \forall k$ et que $P=\sum_{k=0}^{C-1} P_{k}$.

D'autre part, $K \leq \max _{k}\left\lceil\frac{P_{k}}{r_{k}}\right\rceil$ et est donc inférieur ou égal à $P_{r e f}$. Par conséquent, $K \leq h \times P$. La complexité de nos heuristiques dans le cas d'une plate-forme hétérogène composée de plusieurs grappes est donc au plus supérieure à celle de CPA d'un facteur dépendant du nombre de grappe et du degré d'hétérogénéité de la plateforme $(C \times h)$.

\subsection{Evaluation des algorithmes}

Dans cette section nous comparons les deux algorithmes obtenus (HCPA et SHCPA) à CPA (Radulescu et al., 2001b) et MHEFT (Casanova et al., 2004), une heuristique qui adapte un algorithme d'ordonnancement de tâches séquentielles sur plates-formes hétérogènes au cas de DAGs de tâches parallèles. En vue de comparer CPA à nos algorithmes, nous générons une plate-forme homogène (en termes de vitesse des processeurs) équivalente à la plate-forme hétérogène considérée. Cette plateforme équivalente possède la même structure que la plate-forme initiale mais tous les processeurs ont comme vitesse, la vitesse moyenne des processeurs de la plate-forme hétérogène.
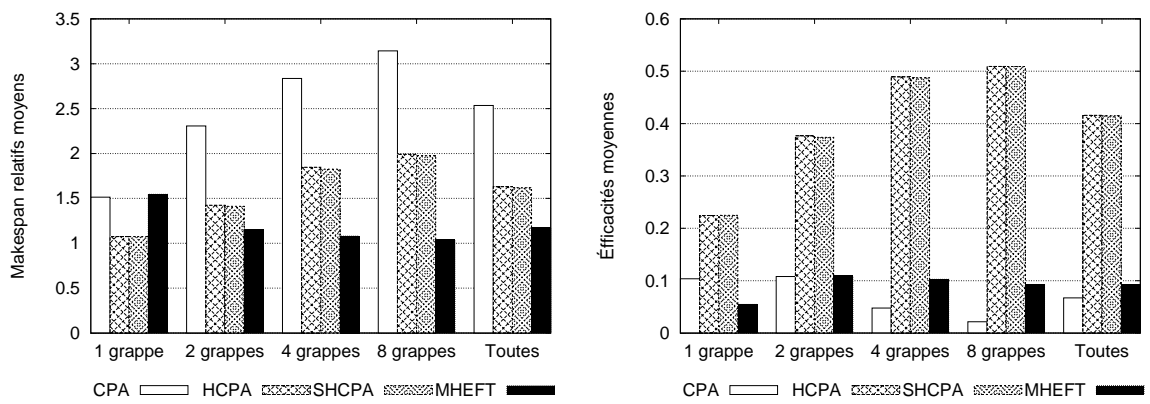

Figure 9. Impact du nombre de grappes

La figure 9 montre les performances moyennes des algorithmes en fonction $\mathrm{du}$ nombre de grappes $(1,2,4$ ou 8) et sur la totalité des plates-formes générées (toutes). En regardant les moyennes sur l'ensemble des plates-formes, nous observons que nos deux heuristiques sont en moyenne plus performantes en termes de makespan par rapport à CPA bien que cette dernière heuristique, qui ne restreint pas l'exécution d'une tâche à l'intérieur d'une même grappe, soit favorisée par le modèle d'Amdhal, ce modèle ne tenant pas explicitement compte des communications intra-tâches. L'heuristique MHEFT est telle que les tâches prêtes sont placées l'une après l'autre sur les ensembles de processeurs qui minimisent leurs dates de fin d'exécution. Par consé- 
quent, MHEFT ne bénéficie pleinement du parallélisme de tâches que si le nombre de grappes de la plate-forme est supérieur au nombre de tâches concurrentes car chaque tâche utilise la totalité de la grappe choisie. La non-exploitation du parallélisme de tâches par MHEFT au sein d'une même grappe explique les meilleurs makespan obtenus par nos heuristiques sur les plates-formes à une grappe. Plus le nombre de grappes augmente, plus le makespan relatif de MHEFT devient meilleur par rapport à celui de nos deux heuristiques. En revanche, MHEFT utilisant de très nombreuses ressources, ses performances au niveau du makespan se font au détriment de l'efficacité.

Du fait que nos deux heuristiques n'allouent des processeurs supplémentaires qu'aux tâches les plus critiques et que la procédure d'allocation s'arrête plus tôt que celle de CPA (utilisation de $T_{A}^{\prime}$, restriction des allocations à l'intérieur d'une seule grappe), celles-ci utilisent plus rationnellement les ressources comparativement à CPA et MHEFT quel que soit le nombre de grappes de la plate-forme. Cela se traduit par une efficacité beaucoup plus importante pour nos deux heuristiques. Ainsi, sur la totalité des simulations, l'efficacité de HCPA et SHCPA est quatre fois meilleure que celle de MHEFT tandis que MHEFT a un makespan relatif seulement une fois et demie meilleur.

Nous pouvons donc conclure que nos deux nouvelles heuristiques sont complémentaires à MHEFT. Si un utilisateur à accès sans partage à une plate-forme contenant de nombreuses grappes, il peut utiliser MHEFT pour réduire le makespan de l'application. En revanche, sur une plate-forme partagée par de nombreux utilisateurs ou le temps d'utilisation des processeurs peut être « facturé » aux utilisateurs, une utilisation plus rationnelle s'impose. Dans ce cas, HCPA et SHCPA garantissent un bon compromis entre l'utilisation efficace des ressources et la réduction du makespan des applications.
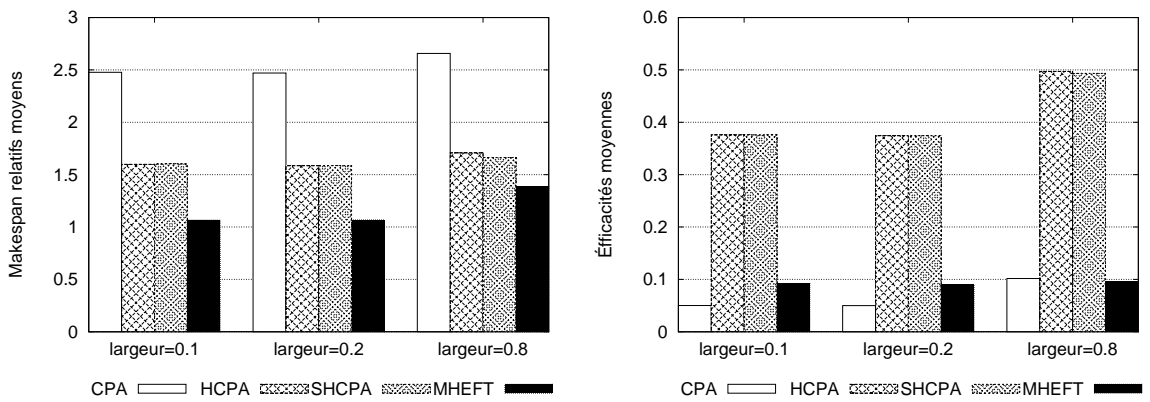

Figure 10. Impact de la largeur des graphes

La figure 10 traduit le fait que le parallélisme de tâche est mieux exploité par HCPA et SHCPA. Pour les DAGs larges (largeur $=0,8$ ), l'écart entre les makespan de MHEFT et ceux de HCPA et SHCPA est réduit tandis que ces dernières fournissent des efficacités nettement meilleures. 

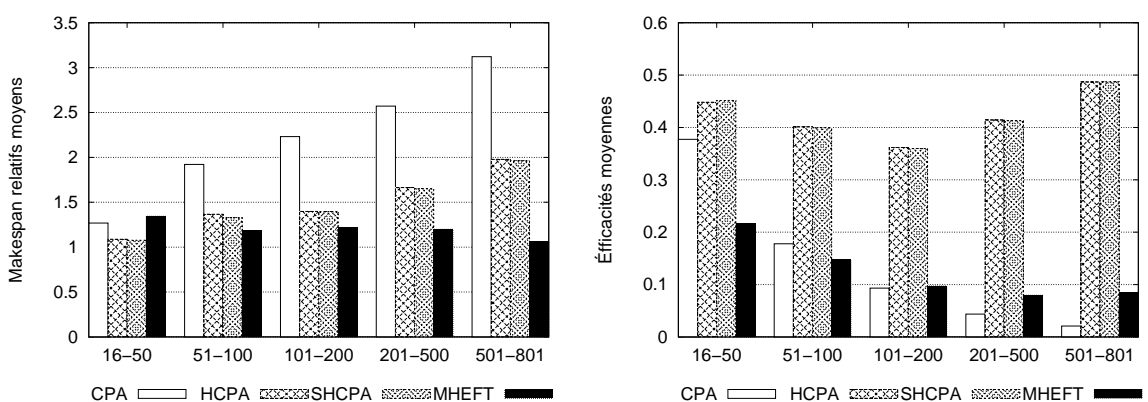

Figure 11. Impact du nombre de næuds des plates-formes

Dans la figure 11, nous avons regroupé les plates-formes en fonction du nombre de processeurs. Pour un nombre de processeurs $P$ compris entre 16 et 50 , tous les DAGs ont leur nombre de tâches $N$ proches de $P$. CPA et HCPA ont alors des performances assez proches aussi bien pour les makespan que pour les efficacités. En revanche, dès que $P$ est très grand par rapport à $N(P \gg N)$, nous pouvons observer l'intérêt de notre contribution en utilisant $T_{A}^{\prime}$ au lieu de $T_{A}$. Celles-ci gardent une bonne efficacité quel que soit le nombre de processeurs. MHEFT utilisant de plus en plus de ressources, son makespan relatif s'améliore au détriment de l'efficacité qui devient très faible par rapport à celles de HCPA et SHCPA.
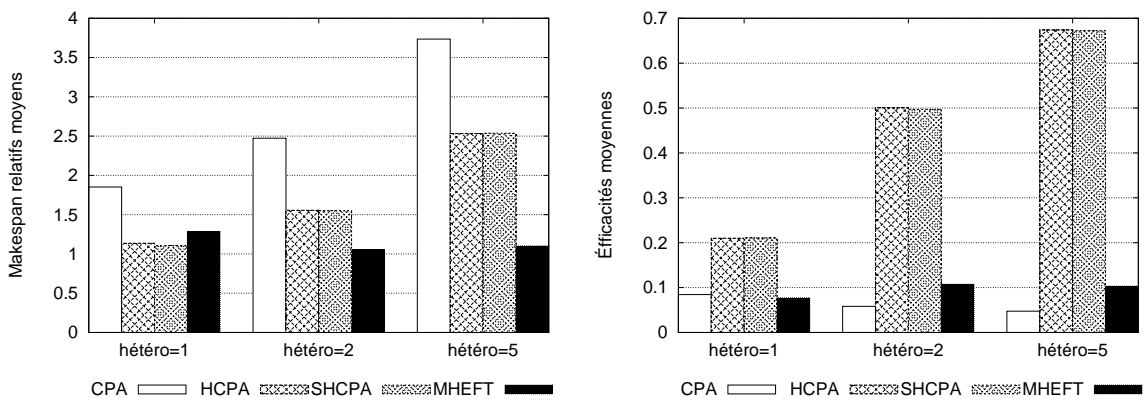

Figure 12. Impact de l'hétérogénéité des plates-formes

Dans la figure 12, lorsque l'hétérogénéité des plates-formes est égale à 1 , nous avons encore une illustration de l'impact de l'utilisation de $T_{A}^{\prime}$. Ces plates-formes sont homogènes en termes de vitesse de processeurs mais peuvent être composées de plusieurs grappes et d'un grand nombre de processeurs. CPA pouvant utiliser des allocations multisites, elle obtient des performances moins bonnes. Lorsque l'hétérogénéité augmente, l'écart se creuse entre nos heuristiques et CPA aussi bien vis-à-vis du makespan que de l'utilisation efficace des ressources. L'allocation d'une tâche sur une grappe dépendant du rapport entre la vitesse des processeurs de cette grappe et 
celle des processeurs de la grappe de référence, HCPA et SHCPA favorisent ainsi l'utilisation des processeurs les plus rapides plutôt que l'utilisation de nombreux processeurs lents. Par conséquent, plus l'hétérogénéité de la plate-forme est élevée, plus nos heuristiques sont efficaces en termes d'utilisation des ressources. MHEFT utilisant au maximum les grappes les plus rapides, elle améliore les temps de complétion par rapport à HCPA et SHCPA mais conserve une efficacité très basse.

\begin{tabular}{|l|c|c|}
\hline Algorithme & Accélération $\perp$ SEQ & Efficacité \\
\hline CPA & 5,48 & $6,72 \%$ \\
\hline HCPA & 7,97 & $41,58 \%$ \\
\hline SHCPA & 8,07 & $41,46 \%$ \\
\hline MHEFT & 9,43 & $9,27 \%$ \\
\hline
\end{tabular}

Tableau 2. Performances relatives par rapport à $S E Q$

Le tableau 2 illustre les performances relatives des heuristiques par rapport au meilleur algorithme d'ordonnancement séquentiel (SEQ) où nous avons calculé les moyennes sur la totalité des simulations. On observe que HCPA et SHCPA sont 4,5 fois plus efficaces que MHEFT tandis que l'accélération moyenne de MHEFT par rapport à SEQ est seulement de 1,2 fois meilleure comparativement à celles nos nouvelles heuristiques. Cela confirme le fait que nos nouvelles heuristiques permettent de trouver un bon compromis entre l'utilisation rationnelle des ressources et la réduction du makespan des applications.

Enfin pour ce qui est de la comparaison entre HCPA et SHCPA, il apparaît que les deux heuristiques donnent des résultats très similaires. Une observation plus fine des résultats de simulations révèle que SHCPA produit un meilleur makespan que HCPA dans 30,96\% des cas et un makespan égal à celui de HCPA dans 44,34\% des cas, HCPA étant meilleur dans les $24,70 \%$ de cas restants. Cela signifie que les heuristiques de liste fondées sur le chemin critique pour le placement des tâches prêtes ne minimisent pas toujours le temps de complétion des applications parallèles. D'autres stratégies peuvent être explorées.

La figure 14 montre les résultats de la simulation des algorithmes sur une grappe de grappes extraite d'une plate-forme réelle. Cette plate-forme est un sous-ensemble de la grille expérimentale grid' 5000 (voir figure 13). Nous avons retenu 6 grappes sur les sites de Lille, Lyon, Nancy, Nice, Orsay et Rennes. Ce sous-ensemble comprend au total 702 processeurs et un réseau relativement rapide dont 5 dorsales à $10 \mathrm{~Gb} / \mathrm{s}$. Sur cette plate-forme de faible hétérogénéité (tous les processeurs ont une vitesse environ égale à $3,3 G$ flops $)$ et où le nombre de processeurs $(P=702)$ est très élevé par rapport au nombre de tâches des applications $(N \leq 50)$, nous constatons que les efficacités de MHEFT et CPA sont très faibles par rapport à HCPA et SHCPA. Or HCPA et SHCPA fournissent des makespan relatifs proches de ceux de MHEFT qui sont en moyenne meilleurs. Cela confirme une fois de plus les bonnes performances de nos deux algorithmes qui obtiennent un meilleur compromis entre l'utilisation des ressources et le temps de complétion des applications. 


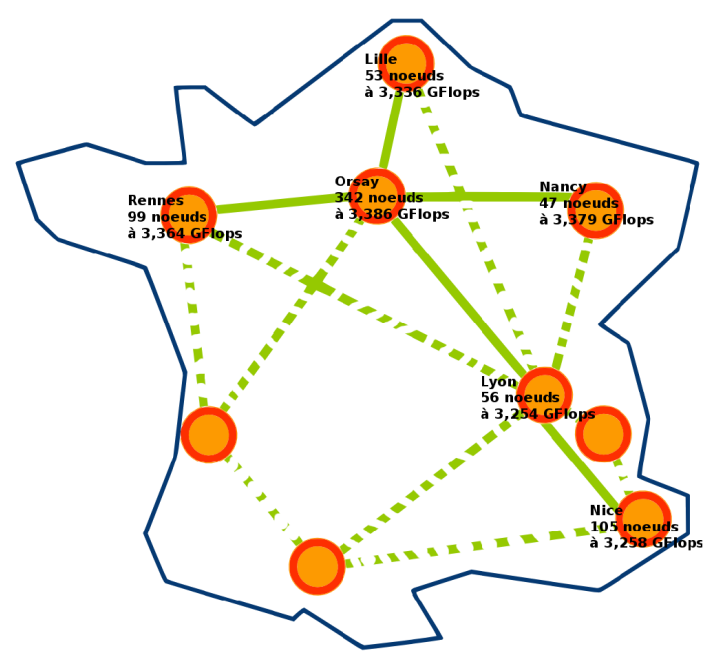

Figure 13. Exemple de grappe de grappes extraite de grid'5000
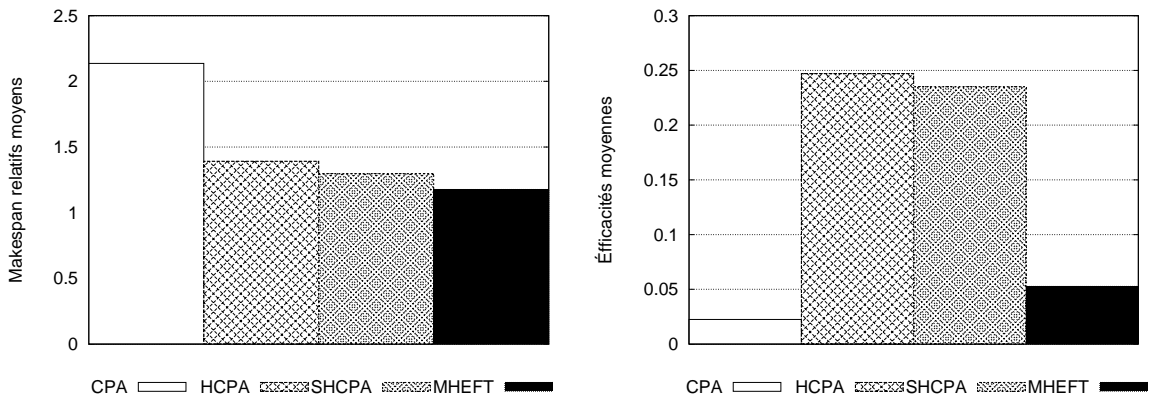

Figure 14. Comparaison sur un sous-ensemble de grid'5000

\section{Conclusion}

Dans cet article, nous avons adapté une heuristique d'ordonnancement de tâches parallèles sur plates-formes homogènes au cas des plates-formes hétérogènes. Pour cela nous avons choisi une heuristique d'ordonnancement en deux étapes de la littérature (CPA (Radulescu et al., 2001b)) dont les performances sont relativement bonnes par rapport aux algorithmes concurrents si on tient compte à la fois de la complexité de ces algorithmes, des temps de complétion obtenus et de l'utilisation des ressources.

Dans un premier temps, nous avons proposé deux améliorations de CPA dans le cas des plates-formes homogènes. La première concerne la phase d'allocation et la seconde, la phase de placement. Dans la phase d'allocation, nous avons redéfini la 
notion d'aire moyenne afin d'arrêter plus tôt la procédure d'allocation de processeurs et de profiter au mieux de l'exécution en parallèle de tâches concurrentes. Nous avons expérimentalement vérifié que cette modification permet en moyenne de réduire le temps de complétion des applications tout en utilisant moins de ressources que CPA. Dans la phase de placement, nous avons mis en place une technique de tassage dont le but est de réduire à la fois le temps d'attente et la date de fin d'exécution d'une tâche prête à placer en diminuant son allocation si cela est nécessaire. Nous avons également vérifié que cette amélioration réduit le temps de complétion des applications par rapport à CPA. De plus, la combinaison de ces deux améliorations fournit un léger gain par rapport à chacune des améliorations prise individuellement, la première amélioration ayant plus d'impact que la seconde.

Ensuite, nous avons adapté l'heuristique obtenue en combinant les deux améliorations au cas de plates-formes hétérogènes de type grappe hétérogène de grappes homogènes. A cet effet, nous avons introduit la notion de grappe de référence qui nous a permis de mieux gérer l'hétérogénéité des plates-formes dans la procédure d'allocation. Le but de cette virtualisation des plates-formes est surtout de conserver une faible complexité pour nos heuristiques. Ainsi, nous avons mis en place deux heuristiques qui se distinguent par leur phase de placement : HCPA et SHCPA. Dans HCPA, nous adaptons la technique de placement de CPA au cas où nous disposons de plusieurs grappes, et donc de plusieurs allocations possibles. La phase de placement de SHCPA est quant à elle basée sur l'heuristique sufferage (Maheswaran et al., 1999).

La complexité de nos deux nouvelles heuristiques est du même ordre que celle de CPA. L'évaluation des heuristiques HCPA et SHCPA en milieu hétérogène révèle qu'elles sont plus performantes que CPA et qu'elles permettent d'obtenir un meilleur compromis entre l'utilisation efficace des ressources et la réduction du temps de complétion des applications par rapport à MHEFT (Casanova et al., 2004).

Dans nos travaux futurs, nous essaierons de mettre en œuvre des heuristiques qui gèrent équitablement l'exécution concurrente de plusieurs DAGs de tâches parallèles sur des agrégations de grappes de calcul. A l'instar de HCPA et SHCPA, ces heuristiques doivent fournir un bon compromis entre la gestion des ressources et les makespan. Pour ces études à venir, nous projetons d'utiliser des plates-formes plus réalistes. Il sera par exemple possible d'avoir des topologies réseau complexes avec des sousgraphes non couplés. Nous prévoyons également l'utilisation de modèles de tâches parallèles qui incluent les coûts des communications intra-tâches.

\section{Remerciements}

Cette étude a été en partie soutenue par l'ARC INRIA OTaPHe, la Région Lorraine et le Gouvernement Ivoirien 


\section{Bibliographie}

Amdahl G., « Validity of the Single Processor Approach to Achieving Large Scale Computing Capabilities », AFIPS 1967 Spring Joint Computer Conference, vol. 30, p. 483-485, April, 1967.

Anderson T. E., Culler D. E., Patterson D. A., « A Case for NOW (Networks Of Workstations) », IEEE Micro, vol. 15, n 1, p. 54-64, 1995.

Boudet V., Desprez F., Suter F., « One-Step Algorithm for Mixed Data and Task Parallel Scheduling Without Data Replication », 17th International Parallel and Distributed Processing Symposium (IPDPS'03), April, 2003.

Casanova H., Desprez F., Suter F., « From Heterogeneous Task Scheduling to Heterogeneous Mixed Parallel Scheduling », 10th International Euro-Par Conference, vol. 3149 of LNCS, Springer, Pisa, Italy, p. 230-237, August, 2004.

Casanova H., Legrand A., Quinson M., « SimGrid : a Generic Framework for Large-Scale Distributed Experimentations », 10th IEEE International Conference on Computer Modelling and Simulation (UKSIM/EUROSIM'08), Cambridge, U.K., April, 2008.

Chrétienne P., « Task Scheduling Over Distributed Memory Machines », Parallel and Distributed Algorithms, North Holland, p. 165-176, 1988.

Dutot P.-F., « Hierarchical Scheduling for Moldable Tasks », 11th International Euro-Par Conference, vol. 3648 of LNCS, Springer, Lisbon, Portugal, p. 302-311, August, 2005.

Lepère R., Trystram D., Woeginger G., « Approximation Algorithms for Scheduling Malleable Tasks Under Precedence Constraints », IJFCS, vol. 13, n 4, p. 613-627, 2002.

Maheswaran M., Ali S., Siegel H. J., Hensgen D., Freund R. F., « Dynamic Matching and Scheduling of a Class of Independent Tasks onto Heterogeneous Computing Systems », 8th Heterogeneous Computing Workshop (HCW'99), IEEE Computer Society, Washington, DC, USA, p. 30, 1999.

Radulescu A., Nicolescu C., van Gemund A., Jonker P., « CPR : Mixed Task and Data Parallel Scheduling for Distributed Systems », 15th International Parallel and Distributed Processing Symposium (IPDPS), April, 2001a. Best Paper Award.

Radulescu A., van Gemund A., « A Low-Cost Approach towards Mixed Task and Data Parallel Scheduling », 15th International Conference on Parallel Processing (ICPP), Valencia, Spain, September, 2001b.

Ramaswamy S., Sapatnekar S., Banerjee P., « A Framework for Exploiting Task and Data Parallelism on Distributed Memory Multicomputers », IEEE Transactions on Parallel and Distributed Systems, vol. 8, n 11, p. 1098-1116, November, 1997.

Rauber T., Rünger G., « Compiler Support for Task Scheduling in Hierarchical Execution Models », Journal of Systems Architecture, vol. 45, p. 483-503, 1998.

Turek J., Wolf J., Yu P., « Approximate Algorihtms for Scheduling Parallelizable Tasks », 4th ACM Symposium on Parallel Algorithms and Architectures, p. 323-332, 1992.

Article reçu le 23 janvier 2007 Accepté après révisions le 15 février 2008 